\title{
Function of Hsf1 in SV40 T-antigen-transformed HEK293T cells
}

\author{
QIYING JIANG $^{1}$, ZHI ZHANG ${ }^{2}$, YANZHONG HU ${ }^{2}$ and YUANFANG MA ${ }^{2}$ \\ ${ }^{1}$ Department of Immunology, Basic Medical College of Zhengzhou University, Zhengzhou, Henan 450052; \\ ${ }^{2}$ Key Laboratory of Cellular and Molecular Immunology, Institute of Immunology, Medical School of Henan University, \\ Kaifeng, Henan 475004, P.R. China
}

Received July 10, 2013; Accepted March 7, 2014

DOI: $10.3892 / \mathrm{mmr} .2014 .2619$

\begin{abstract}
Heat shock factor 1 (HSF1), a main regulator of the heat shock response in eukaryotes, increases cell survival in numerous pathophysiological conditions. The aim of the present study was to o bserve the function of defective HSF1 expression in HEK293T cells. shRNA of human HSF1 was constructed into the retroviral vector pLTHR generating pLTHR-shRNA-HSF1. The shRNA was transiently transfected into HEK293T cells to silence the expression of the HSF1 gene. Cell colony formation, MTT and cell cycle assays were used to analyze the SV40 T-antigen (Ag)-transformed cell proliferation rate. Immunoblotting was used to study the protein expression of HSF1, SV40 T-Ag, p53, p21, heat shock protein 90 (Hsp90), Hsp70 and Hsp25. The results revealed that a deficiency in HSF1 expression inhibited cellular growth. Defective HSF1 upregulated the protein expression of $\mathrm{p} 53$, retinoblastoma protein $(\mathrm{Rb})$ and $\mathrm{SV} 40 \mathrm{~T}-\mathrm{Ag}$, and reduced the association between SV40 T-Ag and p53/Rb, which resulted in growth inhibition of SV40 T-Ag-transformed cells. In conclusion, HSF1 is involved in the regulation of SV40 T-Ag-induced cell growth and modulates the expression of $\mathrm{p} 53$ and $\mathrm{Rb}$ proteins.
\end{abstract}

\section{Introduction}

Heat shock factor 1 (HSF1) is a member of the HSF family, which includes HSF1, HSF2, HSF3 and HSF4 $(1,2)$. These family members have conserved DNA binding domains that can recognize and bind to the heat shock elements (HSEs) in the promoter of the target gene. HSF1 is the dominant regulator of the heat shock response. Upon heat shock or other stresses, the inactive HSF1 is hyperphosphorylated and translocated into the nucleus, forming the active homotrimmerized HSF1. The active form of HSF1 can initiate heat shock protein transcription by binding to the HSEs. The HSF1-mediated heat shock response not only protects cells from environmental or intracellular

Correspondence to: Professor Yuanfang Ma, Key Laboratory of Cellular and Molecular Immunology, Institute of Immunology, Henan University School of Medicine, North Jinming Road, Kaifeng, Henan 475004, P.R. China

E-mail: mayuanfangdoctor@yeah.net

Key words: Hsf1, heat shock proteins, SV40 T-ag, p53, pRb stresses, but is also in involved in the regulation of numerous pathological processes, such as inflammation and tumorigenesis $(3,4)$.

Accumulating evidence demonstrates that HSF1 plays important roles in the regulation of tumorigenesis and development. HSF1 and its relevant downstream targets, heat shock proteins, are elevated in the majority of types of cancer tissue (5-14). Deletion of HSF1 can inhibit DMBA-induced skin cancer, p53 mutant-induced lymphoma and DEN-induced hepatocellular carcinoma. Inhibition of the chaperone activity of heat shock protein 90 (Hsp90) can induce tumor cell apoptosis $(15,16)$. This evidence suggests that HSF1 and its mediated heat shock response can stabilize the homeostasis of highly proliferative tumor cells.

Since HSF1 has been found to be associated with p53 and Ras oncogene-induced tumorigenesis, its roles in the viral oncogene-induced tumorigenesis remain unclear. SV40 is a double-stranded DNA tumor virus. SV40 virus has been isolated from HIV-positive non-Hodgkin lymphoma patient tissues (17). SV40 can express two early oncoproteins; small $\mathrm{t}$-antigen ( $\mathrm{t}-\mathrm{Ag}$ ) and large $\mathrm{T}$ antigen (T-Ag). T-Ag has been used to create a transgenic tumor mouse model and to transform cells for in vitro study (18). T-Ag can transform numerous cell types by interacting with and disrupting the tumor suppression activities of p53 and $\mathrm{Rb}(19,20)$.

In the present study, a cell colony formation assay, an MTT assay and flow cytometry were used to analyze cell proliferation in SV40 T-Ag-expressing cells. To study the mechanisms of HSF1 in SV40 T-Ag-expressing cells, western blotting and an immunoprecipitation assay were used to observe the expression of HSF1, SV40 T-Ag, p53, p21, retinoblastoma protein (Rb), Hsp90, Hsp70 and Hsp25 proteins. HSF1 genes were silenced using an shRNA technique. The present study provides a theoretical basis for the application of HSF1 as a novel target protein to treat various malignant disorders.

\section{Material and methods}

Cell lines and culture. HEK293T cells (referred to as 293T cells; SV40 T-Ag-expressing) and H1299 cells (p53-negative) were obtained from American Type Culture Collection (CRL-6253; Mannassas, VA, USA) and cultured in Dulbecco's modified Eagle's medium (DMEM; Gibco-BRL, Carlsbad, CA, USA) supplemented with $10 \%$ heat-inactivated fetal bovine serum (FBS; Gibco-BRL), $100 \mathrm{U} / \mathrm{ml}$ penicillin and $100 \mu \mathrm{g} / \mathrm{ml}$ 
streptomycin (Gibco-BRL). The morphological characteristics of cells were observed using an inverted microscope (TS100; Nikon, Tokyo, Japan).

All of the above cells and the plasmids were preserved by the Henan University School of Medicine of Cellular and Molecular Immunology Laboratory (Henan, China).

Plasmid construction. A scramble shRNA plasmid, shRNA-HSF1 plasmid and SV40 T-Ag plasmid (Shanghai Shengong Co., Ltd., Shanghai, China) were established in the present study. shRNA of human HSF1 was constructed into the retroviral vector $\mathrm{pLTHR}$ generating the pLTHR-shRNA-HSF1. The shRNA sequence was cloned into the retroviral vector plasmid pLTHR downstream of the U6 promoter. The recombinant plasmid was termed pLTHR-shRNA-HSF1 or, in short, shRNA-HSF1. shRNA of green fluorescent protein cDNA was used as the scramble shRNA. The sequences of the human HSF1 gene were as follows: Forward, 5'-GATCCGTGGACTCCAACCTGGATAATTCAAGAGATTATCCAGGTTGGAGT CCATATTTTTTGGAAg-3'; reverse, 5'-tcgacTTCCAAAAA ATATGGACTCCAACCTGGATAATCTCTTGAATTATCCAGGTTGGAGTCCAC G-3'.

shRNA-HSF1 transfection of 293T cells. shRNA of human HSF1 was constructed into the retroviral vector pLTHR generating the pLTHR-shRNA-HSF1. The shRNA was transiently transfected into 293T cells to silence the expression of HSF1. Transfection was performed using Lipofectamine 2000 (Invitrogen Life Technologies, Carlsbad, CA, USA) and scramble shRNA-transfected 293T cells were used as the control group. Complete medium was employed $6 \mathrm{~h}$ later and cultivation was continued for 48-72 $\mathrm{h}$, and then the cells were harvested by digesting with the trypsin/EDTA (Tiangen Biotech (Beijing) Co., Ltd., Beijing, China) and obtaining the cell deposits. Western blotting was performed to detect the expression of HSF1.

shRNA-HSF1 and SV4O T-Ag co-transfection into H1299 cells. HSF1 genes in H1299 cells were silenced using the abovementioned shRNA technique, and SV40 T-Ag genes were also transfected into the H1299 cells. shRNA-HSF1+SV40 T-Ag was transiently transfected into the H1299 cells using Lipofectamine 2000, and H1299 cells transfected with scramble shRNA+SV40 T-Ag were used as the controls. Complete medium was employed $6 \mathrm{~h}$ later and cultivation was continued for 48-72 h, prior to harvesting. Western blotting was performed to detect the expression of HSF1 and SV40 T-Ag.

MTT assay to examine cell growth. The $293 \mathrm{~T}$ cells (shRNA-HSF1- and scramble shRNA-transfected) were suspended in DMEM medium containing 10\% FBS (Hyclone, Novato, CA, USA). A total of $200 \mu \mathrm{l}$ of the medium containing 800 cells was plated into six 96 -well plates. Each plate possessed all two cell types (including shRNA-HSF1-transfected cells and scramble shRNA cells), which consisted of $200 \mu 1$ DMEM only, and all groups had seven after holes. The cells were cultivated in $20 \mu \mathrm{l}$ MTT. Incubation was continued for $4 \mathrm{~h}$ and then the supernatant was discarded, prior to the addition of $150 \mu \mathrm{l}$ dimethylsulfoxide (Sigma, St. Louis, MO, USA) and $10 \mathrm{~min}$ of oscillation to dissolve the formazan crystals. The light absorption value was examined by Thermo Multiskan Ascent (Nicolet
Evolution 300; Thermo Fisher Scientific, Waltham, MA, USA) at OD490 and then the cell growth curve was drawn with the light absorption (Y-axis) against time (X-axis). The remaining plates were treated using the same procedure.

Cell cycle assay. A total of $1 \times 10^{6} 293 \mathrm{~T}$ cells (shRNA-HSF1and scramble shRNA-transfected, respectively) were collected using three tubes for each type of cells at the different points of the cell cycle. The cells were washed twice with PBS, fixed overnight in precooled $70 \%$ alcohol and then washed twice with precooled PBS. Subsequently, the cells were resuspended in $500 \mu \mathrm{l}$ propidium iodide solution (Sigma; containing $50 \mu \mathrm{g} / \mathrm{ml}$ RNase-A) and incubated for $30 \mathrm{~min}$ in the dark at room temperature. Following this, the quantity of DNA was detected using flow cytometry (FACS Calibur; BD Biosciences, San Jose, CA, USA).

Flat colony-forming assay. The 293T cells (shRNA-HSF1and scramble shRNA-transfected) were cultured for $24 \mathrm{~h}$ and adjusted to a final concentration of $1 \times 10^{3}$ cells $/ \mathrm{ml}$ in DMEM, following digestion by pancreatin. A total of $200 \mu \mathrm{l}$ of the solution was plated into six-well plates and all wells were refilled to $10 \mathrm{ml}$ with DMEM containing 10\% FBS. All of the plates were kept in a humidified atmosphere containing $5 \% \mathrm{CO}_{2}$ at $37^{\circ} \mathrm{C}$ for 2 weeks. The incubation was terminated when visible clones appeared. The plates were washed twice with PBS, fixed by $5 \mathrm{ml}$ of carbinol for $15 \mathrm{~min}$, stained by hematoxylin for $10 \mathrm{~min}$, washed by water to return blue, dried in air and then the visible clones were counted.

Western blotting. The 293T (shRNA-HSF1- and scramble shRNA-transfected) and H1299 (shRNA-HSF1+SV40 T-Agand scramble shRNA+SV40 T-Ag-transfected) cells were lysed with cell lysis buffer containing protease inhibitors to obtain the total proteins. The protein concentrations were measured and the lysates were separated under 10\% SDS-PAGE gel (Tiangen Biotech (Beijing) Co., Ltd.). The proteins were transferred onto nitrocellulose membranes (Millipore, Billerica, MA, USA), which were blocked with Tris-buffered saline and Tween 20 (Sigma) containing 5\% non-fat dried milk for $1 \mathrm{~h}$ at room temperature. The membranes were incubated with primary mouse monoclonal antibodies, including anti-HSF1, -Hsp25, -Hsp70, -heat shock cognate 70 (Hsc70), -Hsp90, -SV40 T-Ag, -p53, -Rb, -p21 and - $\beta$-actin (Santa Cruz Biotechnology, Inc., Santa Cruz, CA, USA) at $4^{\circ} \mathrm{C}$ overnight. Subsequently, the membranes were incubated with secondary anti-mouse polyclonal antibodies (Santa Cruz Biotechnology, Inc.) for $1 \mathrm{~h}$ and then developed using enhanced chemiluminescence (P0018; Biyuntian Biotechnology Institute, Beijing, China), before being exposed to X-ray film. Images of the bands were captured and then analyzed.

Co-immunoprecipitation assay. The 293T cells (shRNA-HSF1and scramble shRNA-transfected) were lysed in cell lysis buffer to obtain the total proteins. The protein concentrations were measured and prepared to the concentration of $1 \mathrm{mg} / \mathrm{ml}$. The solution was incubated with $10 \mu \mathrm{l}$ of Protein A-agarose beads for $1 \mathrm{~h}$ and centrifuged at 15,984 $\mathrm{x}$ g for $5 \mathrm{~min}$. The supernates were transferred into novel EP tubes with the addition of $2 \mu \mathrm{g}$ of anti-SV40 T-Ag. Equal volumes of rabbit immunoglobulin G 
A

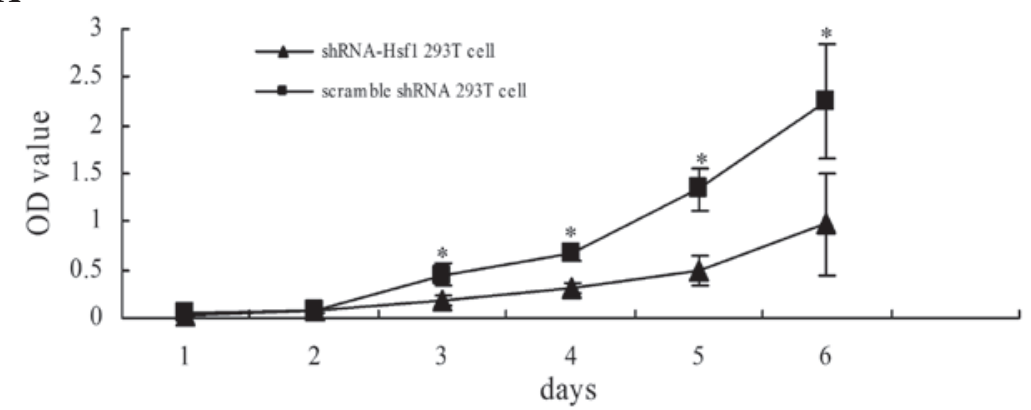

B

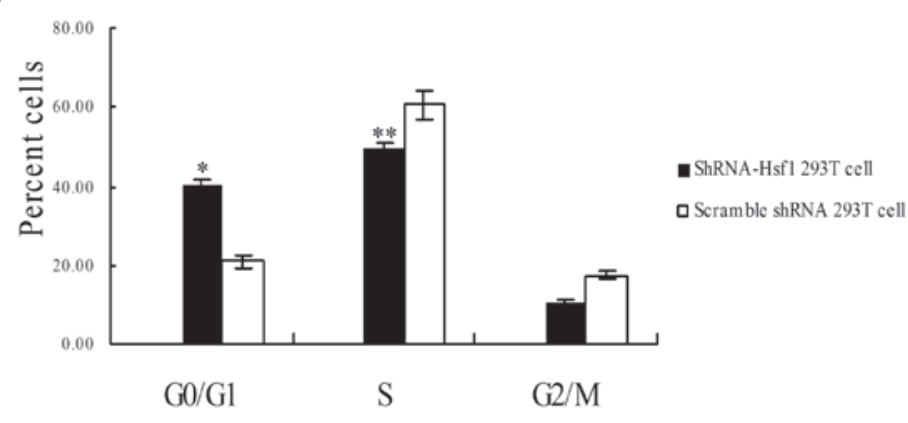

Figure 1. The growth viability of two $293 \mathrm{~T}$ cell lines and the effects of HSF1 on the cell cycle. (A) Growth viability of cells as determined by an MTT assay. ${ }^{*} \mathrm{P}<0.05$, vs. shRNA-HSF1-transfected 293T cells. (B) Effects of HSF1 on the cell cycle distribution. ${ }^{*} \mathrm{P}<0.01$ and ${ }^{* *} \mathrm{P}<0.05$, vs. scramble shRNA-transfected 293 T cells. HSF1, heat shock factor 1; OD, optical density.

were added to the control. All of the samples were shaken overnight at $4^{\circ} \mathrm{C}$, incubated with $40 \mu \mathrm{l}$ of Protein A-agarose beads (Santa Cruz Biotechnology, Inc.) for $2 \mathrm{~h}$ and centrifuged at 15,984 x $\mathrm{g}$ for $5 \mathrm{~min}$, washing away the beads. The Protein A-agarose beads were boiled with $35 \mu \mathrm{l}$ of $2 \mathrm{X}$ loading buffer (Tiangen Biotech (Beijing) Co., Ltd.) and were centrifuged at $15,984 \mathrm{x} g$ for $5 \mathrm{~min}$. The supernates were separated under $10 \%$ SDS-PAGE polyarylamide gel. An immunoprecipitation assay was performed with anti-SV40 T-Ag, -p53, -Rb, -Hsp70 and $\beta$-actin.

Statistical analyses. The data are expressed as the mean \pm standard deviation. Analysis of the gray values of the protein bands obtained by western blotting was performed using Quantity One software (Bio-Rad, Hercules, CA, USA). The optical density values represent the protein expression levels. Statistical analysis of the data was performed using SPSS 13.0 (SPSS, Inc., Chicago, IL, USA). Student's t-test was used for analyzing the paired data that were normally distributed. Comparisons among the values of more than two groups were performed by one-way analysis of variance. $\mathrm{P}<0.05$ was considered to indicate a statistically significant difference.

\section{Results}

Defective HSF1 inhibits 293 T cell proliferation. The MTT assay demonstrated that the growth of the scramble shRNA-transfected $293 \mathrm{~T}$ cells was faster than that of the shRNA-HSF1-transfected 293T cells ( $\mathrm{P}<0.01$; Fig. 1A). These results demonstrated that Hsf1 promotes 293T cell proliferation. The outcomes of the cell cycle assay demonstrated that the percentage of scramble shRNA-transfected cells in G0/G1 and G2/M phase was markedly lower than that of the shRNA-HSF1-transfected cells $(\mathrm{P}<0.01)$. The percentage of
A

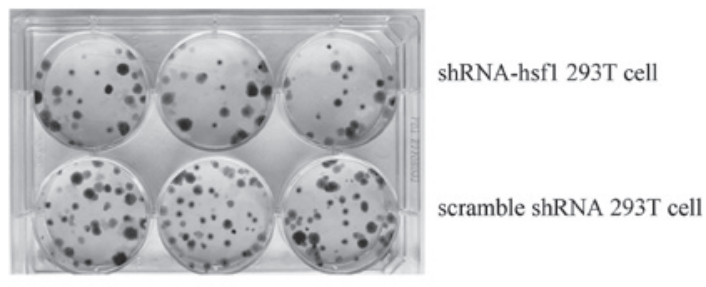

B

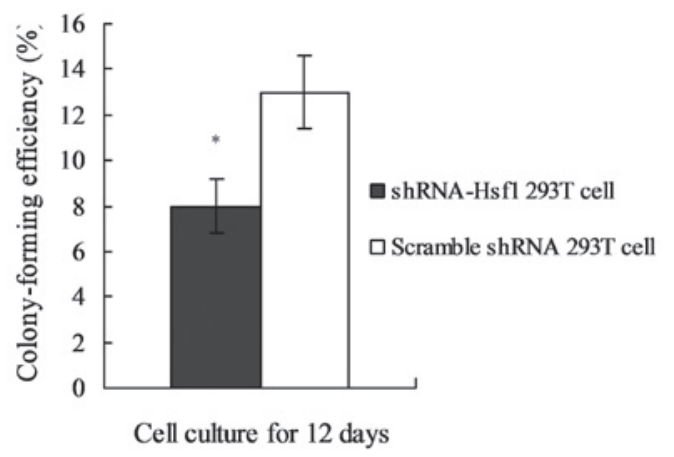

Figure 2. Clone formation of $293 \mathrm{~T}$ cells. (A) Flat cloning assay. (B) Colony-forming efficiency of cell lines, determined by flat cloning assay. ${ }^{*} \mathrm{P}<0.05$, vs. scramble shRNA-transfected 293T cells. HSF1, heat shock factor 1 .

scramble shRNA-transfected cells in $\mathrm{S}$ phase was greater than that of the shRNA-HSF1-transfected cells ( $\mathrm{P}<0.05$; Fig. 1B). These data implied that the scramble shRNA-transfected cells had a stronger proliferation capacity and higher malignancy degree compared with the shRNA-HSF1-transfected cells.

The clone formation rate of the scramble shRNA-transfected 293T cells and the shRNA-HSF1-transfected 293T cells 
A
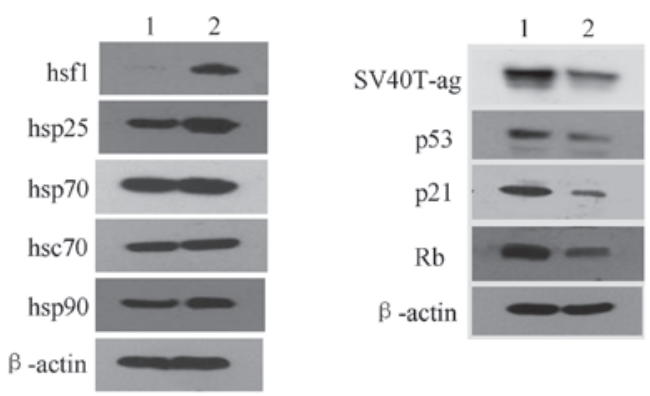

1: shRNA-hsf1 293T cell

2: scramble shRNA 293T cell

B

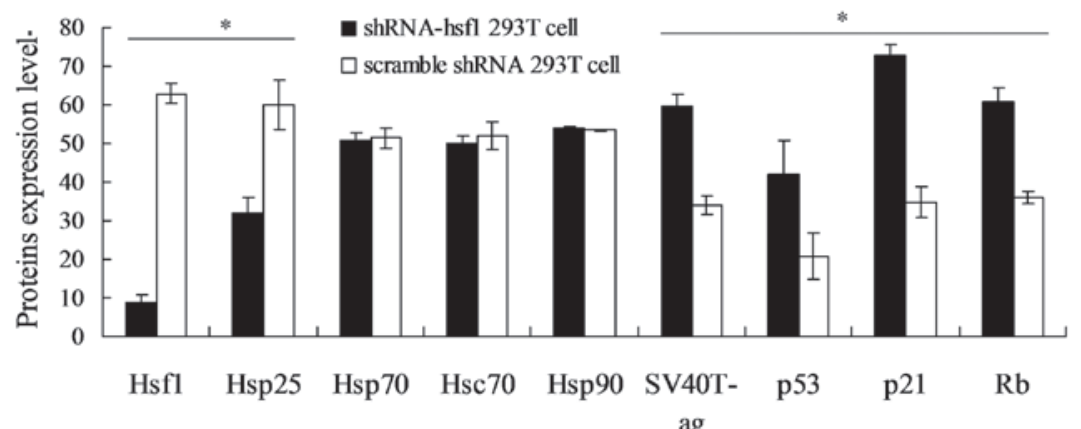

ag

Figure 3. Protein expression was investigated in 293T cells by western blotting. (A) Western blotting and (B) statistical analysis of the expression levels of the proteins. "P<0.05; shRNA-HSF1-transfected 293T cells vs. scramble shRNA-transfected 293T cells. HSF1, heat shock factor 1; Hsp, heat shock protein; Hsc, heat shock cognate; T-Ag, large T-antigen; $\mathrm{Rb}$, retinoblastoma protein.

A

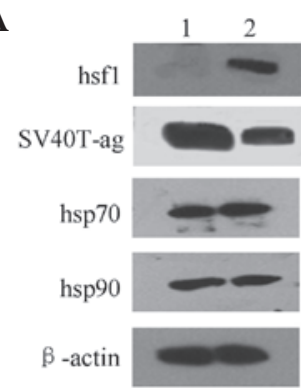

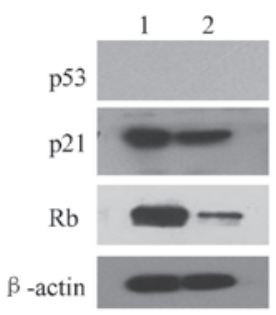

1: H1299 cell (shRNA-Hsfl+SV40T-ag)

2: H1299 cell (scramble shRNA+SV40T-ag)

B

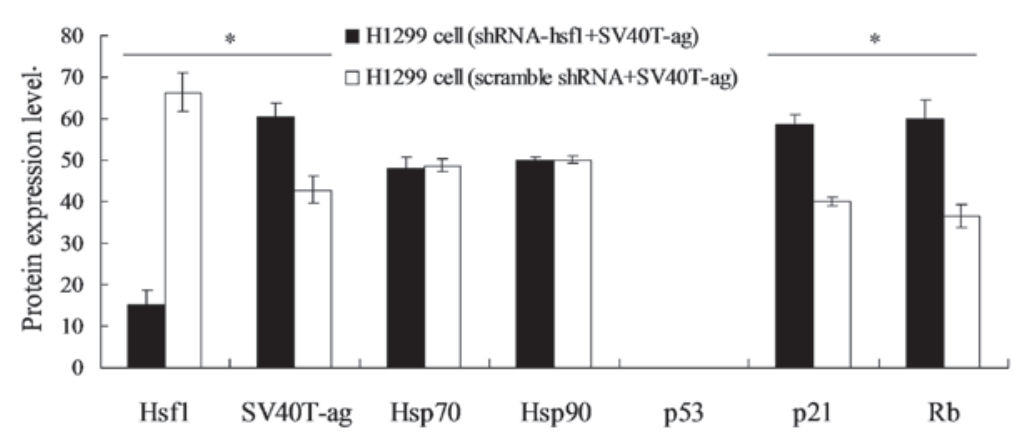

Figure 4. Protein expression was investigated in H1299 cells by western blotting. (A) Western blotting and (B) statistical analysis of the expression levels of the proteins. "P<0.05; shRNA-HSF1+SV40 T-Ag-transfected H1299 cells vs. scramble shRNA+SV40 T-Ag-transfected H1299 cells. HSF1, heat shock factor 1; T-Ag, large T-antigen; Hsp, heat shock protein; Rb, retinoblastoma protein.

was $16.25 \pm 0.524$ and $8.93 \pm 1.711 \%$, respectively. The formation rate of the scramble shRNA-transfected cells was greater than that of the shRNA-HSF1-transfected cells $(\mathrm{P}<0.001$; Fig. 2). This implied that the scramble shRNA-transfected cells had stronger proliferation capacity and higher malignancy degree compared with the shRNA-HSF1-transfected cells.
HSF1 is involved in p53, Rb, p21, Hsp25 and SV40 T-Ag expression. In order to determine the regulatory roles of HSF1 on p53 and Rb, the expression of HSF1 in 293T cells. The western blotting results demonstrated that Rb, p53, p21, Hsp25 and SV40 T-Ag expression was upregulated in the HSF1-silenced cells compared with the scramble shRNA-transfected cells (Fig. 3). However, no change was observed in the expression of 


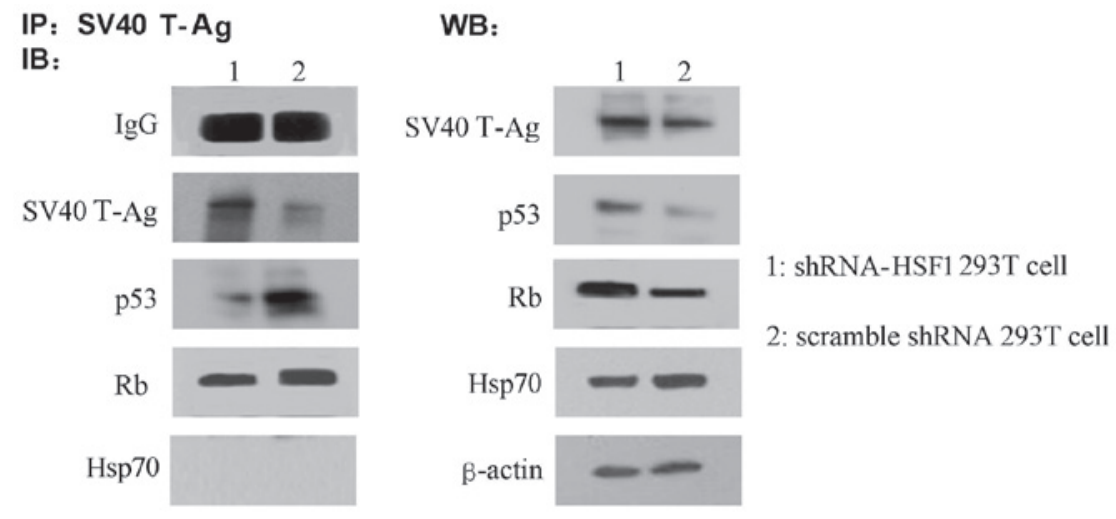

Figure 5. Protein expression was investigated in 293T cells by co-immunoprecipitation. The left image shows immunoprecipitation and the right image shows western blot analysis. IP, immunoprecipitation; IB, immunoblot; WB, western blot; IgG, immunoglobulin G; T-Ag, large T-antigen; Rb, retinoblastoma protein; Hsp7-, heat shock protein 70; HSF1, heat shock factor 1.

Hsp70, Hsp90 and Hsc70 following the silencing of HSF1 in 293T cells (Fig. 3). The results suggest that HSF1 is involved in regulation of the protein expression of $\mathrm{Rb}, \mathrm{p} 53$, and SV40 T-Ag.

HSF 1 regulates $S V 40 T-A, R b$ and $p 21$ protein expression. To investigate the correlations among $\mathrm{p} 53, \mathrm{Rb}$ and SV40 T-Ag, shRNA-HSF1 and SV40 T-Ag were transiently transfected into H1299 cells. H1299 cells are p53-deficient cells, which further facilitated the determination of the effect of HSF1 on $\mathrm{Rb}$ and SV40 T-Ag. The results indicated that silencing of HSF1 in H1299 cells caused an upregulation of Rb and SV40 T-Ag protein expression. However, the expression of Hsp70 and Hsp90 was not significantly different between the two cell types (scramble shRNA+SV40 T-Ag- and shRNA-HSF1+SV40 T-A g-transfected). Additionally, p53 was not detected in either of these two cell types (Fig. 4). These results demonstrated that $\mathrm{HSF} 1$ regulates SV40 T-Ag, Rb and p21 protein expression.

SV40 T-Ag binds to p53 and Rb in scramble shRNA 293 T cells. SV40 T-Ag is the onocoprotein that binds to p53 and Rb leading to cell transformation $(19,20)$. p53, Rb and SV40 T-Ag are all upregulated in HSF1-silenced cells, suggesting a disruption in the interaction among the three proteins. The present immunoprecipitation results indicate that the interactions between SV40 T-Ag and p53, and between SV40 T-Ag and Rb, were weak in shRNA-HSF1-transfected 293T cells compared with the scramble shRNA 293T-transfected cells. In addition, no SV40 T-Ag binding Hsp70 conjugates were identified in the two cell types (Fig. 5).

\section{Discussion}

HSF1, a main regulator of the heat shock response in eukaryotes, can increase the cell survival in a number of pathophysiological conditions. HSF1 not only regulates the expression of Hsps and stress proteins, but also modulates the expression of numerous other non-Hsp genes, which affect multiple biological functions of organisms. The activity of HSF1 is a major factor that regulates the expression of Hsps in tumor tissues, and its over expression is detected in numerous malignant tumor tissues, in which it regulates the development and metastasis of tumor cells by orchestrating a network of different signaling pathways (15).
SV40 induces normal mammalian cell tumorigenesis through SV40 T-Ag, which is a production of viral cancer genes. When SV40 T-Ag binds to p53 and Rb the functions of p53 and $\mathrm{Rb}$ are deactivated, as is the activation of $\mathrm{p} 21$, which facilitates malignant transformation $(20,21)$. The involvement of HSF1 in association with SV40 T-Ag in various malignancies remains unclear; thus, exploiting the regulatory mechanisms of HSF1 is of great theoretical significance and practical application value.

HSF1, a potent regulatory factor in tumorigenesis, facilitates tumorigenesis and development by promoting cell malignant transformation (23). Hoang et al (24) first reported the genesis of human prostate cancer associated with high expression of HSF1. In prostate cancer tissue, enhanced expression of HSF1 and Hsp was found to be concordant with a higher degree of malignancy. It has been demonstrated that using shRNA interference technology to silence HSF1 can significantly reduce the survival of different human cancer cell lines, including breast cell lines (15). However, the use of shRNA interference technology to silence HSF1 in normal cells has little effect on cell growth and survival. This indicates that tumor cells depend on HSF1 more than normal cells, and that HSF1 may promote tumor formation and development $(6,7)$.

The present study demonstrated that $\mathrm{HSF}^{+/+}$ scramble shRNA-transfected 293T cells grew faster than shRNA-HSF1-transfected 293T cells (Student's t-test, $\mathrm{P}<0.001)$. Furthermore, the cloning efficiency of $\mathrm{HSF}^{+/+}$ scramble shRNA-transfected cells was improved compared with that of the shRNA-HSF1-transfected cells (T-test, $\mathrm{P}<0.01)$. These results indicated that the absence of HSF1 inhibited 293T cell proliferation. These results are consistent with previous data, similarly revealing that HSF1 enhances $293 \mathrm{~T}$ cell growth and survival $(6,7)$. The cell cycle comprises of the mitotic phase (M) and interkinesis (G1, S and G2) on the basis of the quantity of DNA in cells. It is stated that the G2 phase, $\mathrm{S}$ phase and $\mathrm{M}$ phase reflect the proliferative activity of cells. In the study of tumor pathology, the $\mathrm{S}$ phase fraction is usually the standard to determine tumor proliferation rate (25). In the present study, cell cycle analysis was performed and it was identified that $\mathrm{HSF}^{+/+}$scramble shRNA-transfected 293T cells had a larger proportion of cells in the $\mathrm{S}$ and $\mathrm{G} 2$ phases compared with that of shRNA-HSF1-transfected 293T cells $(\mathrm{P}<0.05)$. The scramble shRNA-transfected cells had a smaller 
proportion of cells in the G1 phase compared with that of HSF1-silenced cells $(P<0.01)$. These results indicated that the scramble shRNA-transfected cells had a greater proliferative capacity than that of the HSF1-silenced cells.

SV40 T-Ag is capable of combining with p53 and Rb, which induces cell transformation of cancer proteins $(21,25)$. Our results indicated that defective Hsf1 inhibited the SV40T-ag-transformed cell growth and resulted in cell cycle G1/G0 arrest. Therefore, it was hypothesized that HSF1 may be associated with the p53 and Rb signal pathway. The present study revealed an increased expression of SV40 T-Ag, $\mathrm{p} 53, \mathrm{Rb}$ and $\mathrm{p} 21$ proteins in shRNA-HSF1-transfected 293T cells as compared with that in scramble shRNA-transfected 293 T cells. In addition, the p53-negative H1299 cells were used, which were transfected with SV40-Ag simultaneously to HSF1-silencing. When observing the correlation among HSF1, p53 and Rb, the results also demonstrated that HSF1 is associated with the $\mathrm{p} 53$ and $\mathrm{Rb}$ signaling pathway.

In HSF1-silenced cells, p53, Rb and SV40 T-Ag protein expression was upregulated. This suggested that there was disruption in the interaction among the three proteins. The immunoprecipitation experimental results identified that in the scramble shRNA-transfected 293T cells, the interactions between SV40 T-Ag and p53 protein, and between SV40 T-Ag and $\mathrm{Rb}$ protein, were relatively enhanced and the cells grew more rapidly. However, in the shRNA-HSF1-transfected 293T cells, the interactions between SV40 T-Ag and p53 protein, and between SV40 T-Ag and Rb protein, were relatively weak and the cell growth was slower.

In conclusion, silencing of HSF1 resulted in the upregulation of SV40 T-Ag, Rb, p53 and p21 protein expression, the disruption of binding between SV40 T-Ag and $\mathrm{p} 53 / \mathrm{Rb}$ protein, and the inhibition of SV40 T-Ag-induced 293T cell growth. The results suggest that HSF1 promotes the SV40 T-Ag-induced cell proliferation. Furthermore, defective HSF1 causes p53 and $\mathrm{Rb}$ protein accumulation, which may be correlated with the slow growth of shRNA-HSF1 in 293T cells. The results demonstrate that HSF1 is involved in the regulation of SV40 T-Ag-induced cell growth and modulates the expression of $\mathrm{p} 53$ and $\mathrm{Rb}$ proteins.

\section{Acknowledgements}

This study was supported by the national natural science fund (grant no. 30971508 and 30871299 ).

\section{References}

1. He H, Soncin F, Grammatikakis N, Li Y, Siganou A, Gong J, Brown SA, Kingston RE and Calderwood SK: Elevated expression of heat shock factor (HSF) 2A stimulates HSF1-induced transcription during stress. J Biol Chem 278: 35465-35475, 2003.

2. Voellmy R: On mechanisms that control heat shock transcription factor activity in metazoan cells. Cell Stress Chaperones 9: 122-133, 2004.
3. Whitesell L and Lindquist SL: HSP90 and the chaperoning of cancer. Nat Rev Cancer 5: 761-772, 2005.

4. Zhao YJ, Wang H, Liu X, Sun M and Kazuhiro H: Protective effects of glutamine in a rat model of endotoxemia. Mol Med Rep 6: 739-744, 2012.

5. Sherman M and Multhoff G: Heat shock proteins in cancer. Ann NY Acad Sci 1113: 192-201, 2007.

6. Park HK, Cho AR, Lee SC and Ban JY: MPTP-induced model of Parkinson's disease in heat shock protein 70.1 knockout mice. Mol Med Rep 5: 1465-1468, 2012.

7. Sidera K and Patsavoudi E: Extracellular HSP90: conquering the cell surface. Cell Cycle 7: 1564-1568, 2008.

8. Yamauchi T, Adachi S, Yasuda I, Nakashima M, Kawaguchi J, Yoshioka T, Hirose Y, Kozawa O and Moriwaki H: Ultra-violet irradiation induces apoptosis via mitochondrial pathway in pancreatic cancer cells. Int J Oncol 39: 1375-1380, 2011.

9. Lee AS: GRP78 induction in cancer: therapeutic and prognostic implications. Cancer Res 67: 3496-3499, 2007.

10. Razorenova OV, Agapova LS, Budanov AV, Ivanov AV, Strunina SM and Chumakov PM: Retroviral reporter systems for assessing the activity of stress-inducible signal transduction pathways controlled by the p53, HIF-1, and HSF-1 transcription factors. Mol Biol (Mosk) 39: 286-293, 2005 (In Russian).

11. Tang D, Khaleque MA, Jones EL, Theriault JR, Li C, Wong WH, Stevenson MA and Calderwood SK: Expression of heat shock proteins and heat shock protein messenger ribonucleic acid in human prostate carcinoma in vitro and in tumors in vivo. Cell Stress Chaperones 10: 46-58, 2005.

12. Cen H, Zhang S, Fang YM, Tang XP and Dong Q: Induction of HSF1 expression is associated with sporadic colorectal cancer. World J Gastroenterol 10: 3122-3126, 2004.

13. Zhang M, Wang X, Wang X, Hou X, Teng P, Jiang Y, Zhang L, Yang X, Tian J, Li G, Cao J, Xu H, Li Y and Wang Y: Oxymatrine protects against myocardial injury via inhibition of JAK2/STAT3 signaling in rat septic shock. Mol Med Rep 7: 1293-1299, 2013.

14. Khaleque MA, Bharti A, Gong J, Gray PJ, Sachdev V, Ciocca DR, Stati A, Fanelli M and Calderwood SK: Heat shock factor 1 represses estrogen-dependent transcription through association with MTA1. Oncogene 27: 1886-1893, 2008.

15. Dai C, Whitesell L, Rogers AB and Lindquist S: Heat shock factor 1 is a powerful multifaceted modifier of carcinogenesis. Cell 130: 1005-1018, 2007.

16. Wu D, Tao J, Ding J, Qu P, Lu Q and Zhang W: Interleukin-11, an interleukin-6-like cytokine, is a promising predictor for bladder cancer prognosis. Mol Med Rep 7: 684-688, 2013.

17. Sweet BH and Hilleman MR: The vacuolating virus, S.V.40. Proc Soc Exp Biol Med 105: 420-427, 1960.

18. Goodsell DS: The molecular perspective: simian virus 40. Stem Cells 18: 301-303, 2000.

19. Sullivan CS and Pipas JM: T antigens of simian virus 40: molecular chaperones for viral replication and tumorigenesis. Microbiol Mol Biol Rev 66: 179-202, 2002.

20. Tevethia MJ and Ozer HL: SV40-mediated immortalization. Methods Mol Biol 165: 185-199, 2001.

21. Sullivan CS and Pipas JM: T antigens of simian virus 40: molecular chaperones for viral replication and tumorigenesis. Microbiol Mol Biol Rev 66: 179-202, 2002.

22. Tevethia MJ and Ozer HL: SV40-mediated immortalization. Methods Mol Biol 165: 185-199, 2001.

23. Solimini NL, Luo J and Elledge SL: Non-oncogene addiction and the stress phenotype of cancer cells. Cell 130: 986-988, 2007.

24. Hong AT, Huang J, Rudra-Ganguly N, Zheng J, Powell WC, Rabindran SK, Wu C and Roy-Burman P: A novel association between the human shock transcription factor 1 (HSF1) and prostate asenocarcinoma. Am J Pathol 156: 857-864, 2000.

25. Davalieva K, Kiprijanovska S, Broussard C, Petrusevska G and Efremov GD: Proteomic analysis of infiltrating ductal carcinoma tissues by coupled 2-D DIGE/MS/MS analysis. Mol Biol (Mosk) 46: 469-480, 2012. 